\title{
The Keller-Segel system on bounded convex domains in critical spaces
}

\author{
Matthias Hieber ${ }^{1} \cdot$ Klaus Kress $^{1} \cdot$ Christian Stinner $^{1}$ \\ Published online: 18 May 2021 \\ (C) The Author(s) 2021
}

Dedicated to Hideo Kozono on the occasion of his 60-th Birthday

\begin{abstract}
Consider the classical Keller-Segel system on a bounded convex domain $\Omega \subset \mathbb{R}^{3}$. In contrast to previous works it is not assumed that the boundary of $\Omega$ is smooth. It is shown that this system admits a local, strong solution for initial data in critical spaces which extends to a global one provided the data are small enough in this critical norm. Furthermore, it is shown that this system admits for given $T$-periodic and sufficiently small forcing functions a unique, strong $T$-time periodic solution.
\end{abstract}

Keywords Keller-Segel system $\cdot$ Convex domains $\cdot$ Critical spaces $\cdot$ Strong periodic solutions

Mathematics Subject Classification 35Q92 - 35K59 - 35B10 - 47F05 - 92C17

\section{Introduction}

Given a bounded domain $\Omega \subset \mathbb{R}^{n}, n \in \mathbb{N}$, with smooth boundary, the classical KellerSegel system

This article is part of the topical collection dedicated to Prof. Hideo Kozono on the occasion of his 60th birthday, edited by Kazuhiro Ishige, Tohru Ozawa, Senjo Shimizu, and Yasushi Taniuchi.

Matthias Hieber

hieber@mathematik.tu-darmstadt.de

Klaus Kress

kress@mathematik.tu-darmstadt.de

Christian Stinner

stinner@mathematik.tu-darmstadt.de

1 Technische Universität Darmstadt, Fachbereich Mathematik, Schlossgarten-Strasse 7,

64289 Darmstadt, Germany 


$$
\begin{cases}u_{t}=\Delta u-\nabla \cdot(u \nabla v), & x \in \Omega, t>0, \\ v_{t}=\Delta v-v+u, & x \in \Omega, t>0, \\ \frac{\partial u}{\partial v}=\frac{\partial v}{\partial v}=0, & x \in \partial \Omega, t>0 \\ u(x, 0)=u_{0}(x), \quad v(x, 0)=v_{0}(x), & x \in \Omega,\end{cases}
$$

has been investigated during the past decades by very many authors. Here $u$ and $v$ represent the density of a cell population and the concentration of a chemoattractant, respectively. In the situation of domains with smooth boundaries, the problem of local and global existence as well as blow-up of solutions are rather well understood, see e.g., the survey articles $[9,10,16]$ and the references therein. In particular, the existence of global, strong solutions in $L^{p}$-settings has been investigated by Hideo Kozono and coworkers in [13-15]. For further results in this context, see [17, 18]. For recent results on the existence of unique, strong periodic solutions, see [8]. All these results deal with the setting of the whole space or domains with smooth boundaries.

The situation is very different when one considers the Keller-Segel system in domains with nonsmooth boundaries, as e.g. Lipschitz domains. It was recently shown by Horstmann, Meinlschmidt and Rehberg [11] that under suitable conditions on the initial values and the geometry of the domain, one nevertheless obtains again the existence of a unique, strong, local solution to the Keller-Segel system.

In this article we study the Keller-Segel system in bounded convex domains and do not assume that the boundary of $\Omega$ is smooth. The situation of bounded convex domains with smooth boundaries was considered before e.g. in [22, 23], as in this setting, due to the Neumann boundary condition, a classical solution to (1.1) satisfies $\frac{\partial|\nabla v|^{2}}{\partial v} \leq 0$ on $\partial \Omega \times(0, \infty)$. The latter property is helpful to establish a priori estimates for the solution to (1.1) which are used to prove global existence results. Here we use the convexity of the domain to study the Keller-Segel system, similarly to [11], in domains with rough boundaries.

In contrast to [11] we investigate the Keller-Segel system within the framework of time-weighted Sobolev space allowing us to prove strong well-posedness results for initial data $u_{0}$ and $v_{0}$ lying in critical spaces. We note that the modified Keller-Segel system

$$
\begin{cases}u_{t}=\Delta u-\nabla \cdot(u \nabla v), & x \in \Omega, t>0, \\ v_{t}=\Delta v+u, & x \in \Omega, t>0, \\ \frac{\partial u}{\partial v}=\frac{\partial v}{\partial v}=0, & x \in \partial \Omega, t>0, \\ u(x, 0)=u_{0}(x), \quad v(x, 0)=v_{0}(x), & x \in \Omega,\end{cases}
$$

is scaling invariant with respect to the scaling

$$
\left(u_{\lambda}, v_{\lambda}\right)(t, x)=\left(\lambda^{2} u, v\right)\left(\lambda^{2} t, \lambda x\right), \quad \lambda>0
$$

and that the spaces

$$
B_{q, p}^{3 / q-1}(\Omega) \times B_{q, p}^{3 / q}(\Omega)
$$

are scaling invariant spaces for the modified Keller-Segel system in $\Omega \subset \mathbb{R}^{3}$. For results on solutions for (1.2) in various scaling invariant spaces we refer to the work of Kozono, Sugiyama and Wachi [13-15]. 
The situation of bounded convex domains is, of course, less general than the framework of Lipschitz domains as considered in [11], howewer, in the situation of bounded convex domains, several main ingredients of the approach for smooth domains, such as knowledge of the domain of the Neumann Laplacian, its maximal $L^{p}$-regularity as well as the mixed derivative theorem carry over to the given situation. This allows allows us in particular to treat the case of initial data lying in critical Besov spaces as stated precisely in Theorem 1.

Given a bounded convex domain $\Omega \subset \mathbb{R}^{n}$, we are secondly interested in the question whether, given periodic functions $f$ and $g$, there exist time periodic strong solutions to the classical Keller-Segel system. More precisely, for time periodic functions $f_{1}$ and $f_{2}$, we consider the classical inhomogeneous Keller-Segel system

$$
\begin{cases}u_{t}=\Delta u-\nabla \cdot(u \nabla v)+f_{1}(t), & x \in \Omega, t>0, \\ v_{t}=\Delta v-v+u+f_{2}(t), & x \in \Omega, t>0, \\ \frac{\partial u}{\partial v}=\frac{\partial v}{\partial v}=0, & x \in \partial \Omega, t>0 .\end{cases}
$$

We prove the existence of a $T$-periodic solution to (1.3), which is unique in the associated maximal regularity space, provided $f=\left(f_{1}, f_{2}\right)$ is $T$-periodic and sufficiently small in an appropriate norm. We will perform our analysis for $f \in L^{p}\left(0, T ; L^{q}(\Omega)\right)$, which corresponds to the weak setting. Here $p, q \in(1, \infty)$ need to satisfy certain conditions described in detail in Section 2. These conditions are essentially due to the mixed derivative theorem (see e.g. [19, Corollary 4.5.10]), allowing to transfer time into space regularity in a very precise sense, and to Sobolev emdeddings.

The strategy of our approach may be described as follows: we first rewrite the KellerSegel systems (1.1) and (1.3) as semilinear evolution equations, respectively, within the $L^{p}$-setting. Maximal time weighted $L^{p}$-regularity estimates as well as the existence of a bounded $H^{\infty}$-calculus for the differential operators involved imply then the existence of a unique, local solution to the Keller-Segel system for initial data in critical spaces.

Secondly, the above maximal $L^{p}$-regularity estimates for the linearization of the semilinear equation allow first for maximal periodic $L^{p}$-solutions for the linear problem by the Arendt-Bu theorem, see [4]. The existence theory for maximal periodic $L^{p}$-solutions for the linear evolution equations was generalized in [8] to the semi- and quasilinear setting. More precisely, for Banach spaces $X$ we consider time-periodic quasilinear problems of the form

$$
\left\{\begin{array}{l}
u^{\prime}(t)+A(u(t)) u(t)=F(t, u(t)), \quad t \in(0,2 \pi), \\
u(0)=u(2 \pi) .
\end{array}\right.
$$

Assuming natural Lipschitz conditions on $A$ and $F$ as well as that $A(0)$ admits maximal periodic $L^{p}$-regularity, the existence of a unique strong periodic solution to (1.4) is established provided $\|F(\cdot, 0)\|_{\mathbb{F}}$ is small enough, where $\mathbb{F}:=L^{p}(0,2 \pi ; X)$.

This article is organized as follows. In Sect. 2 we present the main results concerning the initial value problem as well as strong $T$-periodic solutions to the classical Keller-Segel system. Section 3 presents results on the Laplacian in the strong and weak setting for bounded convex domains. Sections 4 and 5 present the proofs of our main results. 


\section{Preliminaries and main results}

Consider the Keller-Segel system (1.1), where $\Omega \subset \mathbb{R}^{3}$ denotes a bounded convex domain, $v$ the outward unit normal on $\partial \Omega$ and $f$ a given $T$-periodic function. We study the initial value problem (1.1) for critical spaces as well as the existence of time periodic solutions to the Keller-Segel system (1.3).

We start by rewriting the Keller-Segel system (1.1) as an evolution equation in the Banach space $X_{0}:=\left(W^{1, q^{\prime}}(\Omega)\right)^{\prime} \times L^{q}(\Omega)$ for $1 / q+1 / q^{\prime}=1$ with $q, q^{\prime} \in(1, \infty)$ by introducing the operator $A$ and for $w=(u, v)^{T}$ the mapping $F$ as

$$
A:=\left(\begin{array}{cc}
\Delta_{N}^{w} & 0 \\
1 & \Delta_{N}-1
\end{array}\right), \quad F(w):=\left(\begin{array}{c}
-\nabla \cdot(u \nabla v) \\
0
\end{array}\right) .
$$

Here $\Delta_{N}$ and $\Delta_{N}^{w}$ denote the Neumann Laplacian on $L^{q}(\Omega)$ and $W^{-1, q}(\Omega)=W^{1, q^{\prime}}(\Omega)^{\prime}$ equipped with the domains $D\left(\Delta_{N}\right):=\left\{u \in W^{2, q}(\Omega): \partial_{v} u=0\right.$ on $\left.\partial \Omega\right\}$ and $W^{1, q}(\Omega)$, respectively. For details, see Section 3. Setting $X_{1}:=D\left(\Delta_{N}^{w}\right) \times D\left(\Delta_{N}\right)$, we see that $X_{1} \hookrightarrow X_{0}$ is densely embedded and that $A: X_{1} \rightarrow X_{0}$ is bounded.

The Keller-Segel system (1.1) corresponds then to the equation

$$
\left\{\begin{array}{l}
w^{\prime}(t)-A w(t)=F(w(t)), \quad t \in(0, T), \\
w(0)=w_{0} .
\end{array}\right.
$$

For a Banach space $X, 1<p<\infty$, a time weight $\mu \in(1 / p, 1]$, a time interval $J \subset[0, \infty)$, we set

$$
\begin{aligned}
L_{\mu}^{p}(J ; X) & =\left\{u \in L_{l o c}^{1}(J ; X):\left[t \mapsto t^{1-\mu} u(t)\right] \in L^{p}(J ; X)\right\}, \\
H_{\mu}^{1, p}(J ; X) & =\left\{u \in L_{\mu}^{p}(J ; X) \cap H_{l o c}^{1,1}(J ; X): u^{\prime} \in L_{\mu}^{p}(J ; X)\right\} .
\end{aligned}
$$

Here $u^{\prime}$ denotes the time derivative of $u$ in the distributional sense.

We aim for solutions in the maximal regularity space

$$
\mathbb{E}_{\mu}(J):=H_{\mu}^{1, p}\left(J ; X_{0}\right) \cap L_{\mu}^{p}\left(J ; X_{1}\right) .
$$

The associated real interpolation space $X_{\mu-1 / p}$ for the initial data is given by $X_{\mu-1 / p}=\left(X_{0}, X_{1}\right)_{\mu-1 / p, p}$. Since in the given situation of bounded convex domains, the domains of $\Delta_{N}^{w}$ and $\Delta_{N}$ are explicitly known, it follows as in Theorem 5.2 of [2] that

$$
\left(X_{0}, X_{1}\right)_{s, p}=B_{q, p}^{2 s-1}(\Omega) \times{ }_{N} B_{q, p}^{2 s}(\Omega),
$$

where

$$
{ }_{N} B_{q, p}^{2 s}(\Omega)= \begin{cases}\left\{u \in B_{q, p}^{2 s}(\Omega): \partial_{v} u=0 \text { on } \partial \Omega\right\}, & s \in(1 / 2+1 / 2 q, 1), \\ B_{q, p}^{2 s}(\Omega), & s \in(0,1 / 2+1 / 2 q) .\end{cases}
$$

We show in Section 4 that the critical value $\mu_{c}$ of $\mu$ for which we obtain local wellposedness for (1.1) is given by $\mu_{c}=3 / 2 q+1 / p$. It is thus natural to call this value of $\mu$ the critical weight and it is hence meaningful to name $X_{\mu_{c}-1 / p, p}$ the critical space for (1.1).

Our first result on the Keller-Segel system on bounded convex domains reads as follows. 
Theorem 1 Let $\Omega \subset \mathbb{R}^{3}$ be a bounded and convex domain and $p, q \in(1, \infty)$ such that $q \in(3 / 2,2]$ and $3 / 2 q+1 / p \leq 1$. Then, for all $\left(u_{0}, v_{0}\right) \in B_{q, p}^{3 / q-1}(\Omega) \times{ }_{N} B_{q, p}^{3 / q}(\Omega)$ the chemotaxis system (1.1) admits a unique solution $w=(u, v)^{T}$ with

$$
w \in H_{\mu}^{1, p}\left(0, a ; X_{0}\right) \cap L_{\mu}^{p}\left(0, a ; X_{1}\right)
$$

for some $a>0$ and $\mu=3 / 2 q+1 / p$.

Remark 1 It is interesting to compare Theorem 1 with the results obtained in [11] dealing with a much more complex geometrical situation. It shows that the method of time-weights combined with the special situation of bounded convex domains allows to improve the regularity index for the initial data by more than 1 , meaning from $B_{q, r}^{s}(\Omega)$ for $s>3 / q+1$ and $r>2(1-3 / q)^{-1}$ in [11] to $B_{q, p}^{3 / q}(\Omega)$.

In order to extend Theorem 1 to a global existence result for small data, we consider for $1<r<\infty$ the space $L_{0}^{r}(\Omega)=\left\{u \in L^{r}(\Omega): \int_{\Omega} u=0\right\}$ consisting of all $L^{r}$-functions having mean zero. We then set $X_{0}:=\left(W^{1, q^{\prime}}(\Omega) \cap L_{0}^{q^{\prime}}(\Omega)\right)^{\prime} \times L^{q}(\Omega)$ and consider the weak Neumann Laplacian $\Delta_{N, 0}^{w}$ in $\left(W^{1, q^{\prime}}(\Omega) \cap L_{0}^{q^{\prime}}(\Omega)\right)^{\prime}$ as defined in Section 3. Set

$$
A_{0}:=\left(\begin{array}{cc}
\Delta_{N, 0}^{w} & 0 \\
1 & \Delta_{N}-1
\end{array}\right), \quad F(w):=\left(\begin{array}{c}
-\nabla \cdot(u \nabla v) \\
0
\end{array}\right) .
$$

on $X_{0}$ with $X_{1}=D\left(\Delta_{N, 0}^{w}\right) \times D\left(\Delta_{N}\right)$. Note that

$$
\left(X_{0}, X_{1}\right)_{s, p}=B_{q, p}^{2 s-1}(\Omega) \cap L_{0}^{q}(\Omega) \times{ }_{N} B_{q, p}^{2 s}(\Omega) .
$$

Then the general theory of semilinear equations in time-weigted spaces (see e.g. [20, Cor.2.2]) implies the following result.

Corollary 1 Let $\Omega \subset \mathbb{R}^{3}$ be a bounded and convex domain and $p, q \in(1, \infty)$ such that $q \in(3 / 2,2]$ and $3 / 2 q+1 / p \leq 1$. Then there exists $r_{0}>0$ such that the local solution $w$ given in Theorem 1 exists globally and converges exponentially to zero in $\left(X_{0}, X_{1}\right)_{1-1 / p, p}$ provided $\left\|w_{0}\right\|_{\left(X_{0}, X_{1}\right)_{3 / 2 q, p}} \leq r_{0}$.

Our second main result concerns the existence of strong $T$-periodic solutions to the KellerSegel model in bounded convex domains. For recent results on periodic solutions in the situation of bounded domains with smooth boundaries we refer to [8]. Similarly as above, we rewrite the Eq. (1.3) as an evolution equation on $X_{0}:=\left(W^{1, q^{\prime}}(\Omega) \cap L_{0}^{q^{\prime}}(\Omega)\right)^{\prime} \times L^{q}(\Omega)$, where $L_{0}^{r}(\Omega)=\left\{u \in L^{r}(\Omega): \int_{\Omega} u=0\right\}$ for $1<r<\infty$. To this end, we recall $A_{0}$ and define for $w=(u, v)^{T}$ the mapping $H$ as

$$
A_{0}=\left(\begin{array}{cc}
\Delta_{N, 0}^{w} & 0 \\
1 & \Delta_{N}-1
\end{array}\right), \quad H(t, w):=\left(\begin{array}{c}
-\nabla \cdot(u \nabla v)+f_{1}(t) \\
f_{2}(t)
\end{array}\right) .
$$

Here $\Delta_{N}$ and $\Delta_{N, 0}^{w}$ denote the Neumann Laplacian on $L^{q}(\Omega)$ and $\left(W^{1, q^{\prime}}(\Omega) \cap L_{0}^{q^{\prime}}(\Omega)\right)^{\prime}$ equipped with the domains $D\left(\Delta_{N}\right):=\left\{u \in W^{2, q}(\Omega): \partial_{v} u=0\right.$ on $\left.\partial \Omega\right\} \quad$ and 
$W^{1, q}(\Omega) \cap L_{0}^{q}(\Omega)$, respectively. For details, see Section 3. Setting $X_{1}:=D\left(\Delta_{N, 0}^{w}\right) \times D\left(\Delta_{N}\right)$, we see that $X_{1} \hookrightarrow X_{0}$ is densely embedded and that $A_{0}: X_{1} \rightarrow X_{0}$ is bounded.

The Keller-Segel system in the periodic setting corresponds then to the equation

$$
\left\{\begin{array}{l}
w^{\prime}(t)-A_{0} w(t)=H(t, w(t)), \quad t \in(0, T), \\
w(0)=w(T) .
\end{array}\right.
$$

Being interested again in strong solutions, we define

$$
\begin{aligned}
& \mathbb{E}_{1}:=L^{p}\left(0, T ; W^{1, q}(\Omega) \cap L_{0}^{q}(\Omega)\right) \cap W^{1, p}\left(0, T ;\left(W^{1, q^{\prime}}(\Omega) \cap L_{0}^{q^{\prime}}(\Omega)\right)^{\prime}\right), \\
& \mathbb{E}_{2}:=L^{p}\left(0, T ; D\left(\Delta_{N}\right)\right) \cap W^{1, p}\left(0, T ; L^{q}(\Omega)\right),
\end{aligned}
$$

as well as

$$
\mathbb{F}:=L^{p}\left(0, T ; X_{0}\right) \text { and } \mathbb{E}:=\mathbb{E}_{1} \times \mathbb{E}_{2} .
$$

Our result on periodic solutions to (1.3) reads as follows.

Theorem 2 Let $\Omega \subset \mathbb{R}^{3}$ be a bounded convex domain domain, $T>0, p, q \in(1, \infty)$ such that $3 / 2<q \leq 2$ and $3 / 2 q+1 / p<1$ and let $f=\left(f_{1}, f_{2}\right) \in L^{p}\left(0, T ; X_{0}\right)$ be a T-periodic function.

Then there exists $r_{0}>0$ such that for any $r \in\left(0, r_{0}\right)$ there is $\delta=\delta(r)>0$ such that if $\|f\|_{\mathbb{F}}<\delta$, then there exists a T-periodic solution $w=(u, v)^{T} \in \mathbb{E}$ to $(1.3)$, which is unique in $\overline{B_{\mathbb{E}}}(0, r)$.

Remark 2 (Nonnegative solutions). We remark that the solutions obtained in Corollary 1 and Theorem 2 are not nonnegative, as $u(t) \in L_{0}^{q}(\Omega)$ for all $t \geq 0$. Since $u$ and $v$ represent the density of a cell population and the concentration of a chemoattractant, respectively, nonnegative solutions of Keller-Segel systems are of particular interest. Nonnegative solutions can be obtained from Corollary 1 and Theorem 2 by using the approach from [8, Theorem 2.4 and Corollary 2.5]. Given a nonnegative solution to (1.1) or (1.3) (for $f_{1} \equiv 0$ ) with $M:=\int_{\Omega} u_{0} \geq 0,(U, V):=(u-M, v-M)$ is a solution to a modified Keller-Segel system, namely where the first equation in (1.1) or (1.3) is replaced by $u_{t}=\Delta u-\nabla \cdot((u+M) \nabla v)$. The existence proof for the modified system is completely similar to the reasoning in Corollary 1 and Theorem 2, respectively. Then $(u, v)=$ $(U+M, V+M)$ is a nonnegative solution to (1.1) or (1.3), respectively.

\section{The Laplacian on bounded and convex domains}

For bounded domains $\Omega \subset \mathbb{R}^{n}$ with smooth boundary $\partial \Omega$ it is well known that the Laplacian $\Delta$ with domain $D(\Delta)=\left\{u \in W^{2, q}(\Omega): \partial_{v} u=0\right.$ on $\left.\partial \Omega\right\}$ generates an analytic semigroup of positive contractions on $L^{q}(\Omega)$ for all $q \in(1, \infty)$ and that it satisfies the maximal $L^{p}$-regularity property, see [5].

The results by Wood [24] show that this is no longer the case for arbitrary Lipschitz domains. However, under suitable assumptions on the Lipschitz domain $\Omega$ and the exponent $q$, the above operator still generates a positive, analytic and contractive semigroup on $L^{q}(\Omega)$. 
In the following, we consider bounded, convex domains $\Omega \subset \mathbb{R}^{n}, n \geq 2$, and define the Neumann-Laplacian $\Delta_{N}$ on $L^{q}(\Omega)$ for $1<q<\infty$ by

$$
\begin{aligned}
\Delta_{N} u & :=\Delta u, \\
D\left(\Delta_{N}\right) & :=\left\{u \in W^{2, q}(\Omega): \partial_{v} u=0 \text { on } \partial \Omega\right\} .
\end{aligned}
$$

For the particular situation of $n=3$ and $1<q \leq 2$ it was proved by Wood [24] that $\Delta_{N}$ generates an analytic semigroup of positive contractions on $L^{q}(\Omega)$ provided $\Omega$ is bounded and convex. He proved in addition that $-\Delta_{N}$ admits the property of $L^{p}[0, T]$-regularity on $L^{q}(\Omega)$ provided $1<q \leq 2$ and $0<T<\infty$.

Since $\Delta_{N}$ generates a positive semigroup on $L^{q}(\Omega)$, it follows from the results in [6] (see also Section 10.7 of [12]) that $-\Delta_{N}$ admits a bounded $H^{\infty}$-calculus on $L^{q}(\Omega)$ as well as on $L_{0}^{q}(\Omega)$ with $\phi_{-\Delta_{N}}^{\infty} \leq \pi / 2$ provided $\Omega$ is bounded, convex and $n=3$ and $1<q \leq 2$. Since the semigroup generated by $\Delta_{N}-\omega$ is bounded analytic on $L^{q}(\Omega)$ for suitable $\omega \geq 0$, we conclude by Theorem 10.7.13 of [12] that $\phi_{-\Delta_{N}+\omega}^{\infty}<\pi / 2$. This reproves in particular the fact that $-\Delta_{N}$ admits maximal $L^{p}$-regularity on $L^{q}(\Omega)$ provided $\Omega$ is bounded, convex and $1<q \leq 2$.

Since $-\Delta_{N}$ admits a bounded $H^{\infty}$-calculus on $L^{q}(\Omega)$ it follows that

$D\left(\left(-\Delta_{N}\right)^{1 / 2}\right)=W^{1, q}(\Omega)=: Y_{1}$ and that $\left(-\Delta_{N}\right)^{1 / 2}$ admits a bounded $H^{\infty}$-calculus on $Y_{0}:=L^{q}(\Omega)$. Setting $B_{0}:=\left(-\Delta_{N}+1\right)^{1 / 2}$, Theorem V.1.5.1 of [3] implies that $\left(Y_{0}, B_{0}\right)$ defines an interpolation-extrapolation scale $\left(Y_{\alpha}, B_{\alpha}\right)$ for $\alpha \in \mathbb{R}$. In particular, the operator $B_{-1}: Y_{0} \rightarrow Y_{-1}=W^{-1, q}(\Omega)=\left(W^{1, q^{\prime}}(\Omega)\right)^{\prime}$ defines a linear isomorphism provided $\Omega$ is bounded convex and $1<q \leq 2$.

We next introduce the complex interpolation-extrapolation scale $\left(X_{\alpha}, A_{\alpha}\right), \alpha \in \mathbb{R}$, generated by $\left(X_{0}, A_{0}\right)=\left(L^{q}(\Omega), \Delta_{N}\right)$. Consider the weak Neumann-Laplacian $\Delta_{N}^{w}$ on $W^{-1, q}(\Omega)$ defined by

$$
\Delta_{N}^{w}:=A_{-1 / 2}: W^{1, q}(\Omega) \rightarrow W^{-1, q}(\Omega),
$$

which has the explicit representation

$$
<\Delta_{N}^{w}|\phi>=<\nabla u| \nabla \phi>_{L^{2}}
$$

for $(u, \phi) \in W^{1, q}(\Omega) \times W^{1, q^{\prime}}(\Omega)$ with $1 / q+1 / q^{\prime}=1$. Since

$$
\left\|f\left(-\Delta_{N}^{w}\right)\right\|_{\mathcal{L}\left(W^{-1, q}\right)}=\left\|B_{-1} f\left(-\Delta_{N}^{w}\right)\left(B_{-1}\right)^{-1}\right\|_{\mathcal{L}\left(W^{-1, q}\right)} \leq C\left\|f\left(-\Delta_{N}\right)\right\|_{\mathcal{L}(L q)}
$$

for $f \in H_{0}^{\infty}\left(\Sigma_{\varphi}\right)$ it follows that $-\Delta_{N}^{w}$ has a bounded $H^{\infty}$-calculus on $W^{-1, q}(\Omega)$ with the same angle as $-\Delta_{N}$. Here $H_{0}^{\infty}\left(\Sigma_{\varphi}\right)$ and $f\left(-\Delta_{N}^{w}\right)$ are defined as in [5]. In particular, $\Delta_{N}^{w}$ admits maximal $L^{p}$-regularity on $W^{-1, q}(\Omega)$.

Concerning bounded imaginary powers of $\Delta_{N}$ note that the transference principle of Coifman-Weiss implies that $\left\|\left(-\Delta_{N}\right)^{i t}\right\|_{L^{q}} \leq M\left(1+t^{2}\right) e^{\pi|t| / 2}$ for $t \in \mathbb{R}$. Since $\Delta_{N}$ is selfadjoint on $L^{2}(\Omega)$ it follows by interpolation that $-\Delta_{N}$ has bounded imaginary powers on $L^{q}(\Omega)$ of angle $\theta$, where $\theta>\pi|1 / q-1 / 2|$. As before, here $n=3$ and $q \in(1,2]$.

We summarize our calculations in the following proposition.

Proposition 1 Let $p \in(1, \infty), 1<q \leq 2$ and $\Omega \subset \mathbb{R}^{3}$ be bounded convex domain. 
(a) The operator $-\Delta_{N}$ admits a bounded $H^{\infty}$-calculus on $L^{q}(\Omega)$ and there exists $\omega \geq 0$ such that $-\Delta_{N}+\omega$ admits a bounded $H^{\infty}$-calculus on $L^{q}(\Omega)$ of angle $\phi_{-\Delta_{N}+\omega}^{\infty}<\pi / 2$.

(b) The operator $-\Delta_{N}^{w}$ admits a bounded $H^{\infty}$-calculus on $W^{-1, q}(\Omega)$ and there exists $\omega \geq 0$ such that $-\left(\Delta_{N}+\omega\right)^{w}$ admits a bounded $H^{\infty}$-calculus on $W^{-1, q}(\Omega)$ of angle $\phi_{-\left(\Delta_{N}+\omega\right)^{w}}^{\infty}<\pi / 2$.

(c) The operators $-\Delta_{N}$ and $-\Delta_{N}^{w}$ admit bounded imaginary powers on $L^{q}(\Omega)$ and $W^{-1, q}(\Omega)$, respectively, of angle $\varphi>\pi|1 / q-1 / 2|$.

(d) The operators $-\Delta_{N}^{w}$ and $-\Delta_{N}$ admit maximal $L^{p}([0, T])$-regularity on $W^{-1, q}(\Omega)$ and $L^{q}(\Omega)$, respectively.

We also consider the Neumann Laplacian $\Delta_{N, 0}$ on $L_{0}^{q}(\Omega)$ as well as the weak Neumann Laplacian on $\left(W^{1, q^{\prime}}(\Omega) \cap L_{0}^{q^{\prime}}(\Omega)\right)^{\prime}$, where as above $L_{0}^{r}(\Omega)=\left\{u \in L^{r}(\Omega): \int_{\Omega} u=0\right\}$ for $1<r<\infty$ and set

$$
\begin{aligned}
\Delta_{N, 0}^{w} u & :=\Delta_{N}^{w} u, \\
D\left(\Delta_{N, 0}^{w}\right) & :=W^{1, q}(\Omega) \cap L_{0}^{q}(\Omega) .
\end{aligned}
$$

Corollary 2 Let $p \in(1, \infty), 1<q \leq 2$ and $\Omega \subset \mathbb{R}^{3}$ be a bounded and convex domain.

(a) Then there exists $w \geq 0$ such that $-\Delta_{N, 0}+\omega$ admits a bounded $H^{\infty}$-calculus on $L_{0}^{q}(\Omega)$ of angle $\phi_{-\Delta_{N}+\omega}^{\infty}<\pi / 2$ and $-\left(\Delta_{N, 0}+\omega\right)^{w}$ admits a bounded $H^{\infty}$-calculus on $\left(W^{1, q^{\prime}}(\Omega) \cap L_{0}^{q^{\prime}}(\Omega)\right)^{\prime}$ of angle $\phi_{-\left(\Delta_{N, 0}+\omega\right)^{w}}^{\infty}<\pi / 2$.

(b) The operator $-\Delta_{N, 0}^{w}$ admits bounded imaginary powers on $\left(W^{1, q^{\prime}}(\Omega) \cap L_{0}^{q^{\prime}}(\Omega)\right)^{\prime}$ of angle $\varphi>\pi|1 / q-1 / 2|$. In particular, the operator $-\Delta_{N, 0}^{w}$ admits maximal $L^{p}([0, T])$-regularity on $\left(W^{1, q^{\prime}}(\Omega) \cap L_{0}^{q^{\prime}}(\Omega)\right)^{\prime}$.

Remark 3 Consider the operators $A$ and $A_{0}$ defined as in (2.1) and (2.3) on the associated spaces $X_{0}$ with domains $X_{1}$. Then $X_{0}$ is an UMD space and Proposition 1 and Corollary 2 imply that $A$ and $A_{0}$ admit a bounded $H^{\infty}$-calculus on $X_{0}$. The mixed derivative theorem [19, Cor.4.5.10] implies then for $\beta \in(0,1), p \in(1, \infty)$ the embedding

$$
H^{1, p}\left(\mathbb{R} ; X_{0}\right) \cap L^{p}\left(\mathbb{R} ; X_{1}\right) \hookrightarrow H^{1-\beta, q}\left(\mathbb{R} ; X_{\beta}\right),
$$

where $\Omega \subset \mathbb{R}^{3}$ is bounded and convex.

\section{The initial value problem on convex domains}

We start this section by recalling from [21] and [20] (see also Chapter I of [7]) some results on semilinear evolution equations, on which we will base the proof of Theorem 1 .

Let $X_{0}, X_{1}$ be Banach spaces such that $X_{1} \hookrightarrow X_{0}$ is densely embedded, and let $A: X_{1} \rightarrow$ $X_{0}$ be bounded. For $0<T \leq \infty$ consider the semi-linear problem

$$
u^{\prime}+A u=F(u)+f \quad \text { on }(0, T) \quad \text { with } \quad u(0)=u_{0} .
$$


For a Banach space $X$, a time weight $\mu \in(1 / p, 1]$, a time interval $J \subset[0, \infty)$ and $k \in \mathbb{N}$, we set

$$
\begin{aligned}
L_{\mu}^{p}(J ; X) & =\left\{u \in L_{l o c}^{1}(J ; X):\left[t \mapsto t^{1-\mu} u(t)\right] \in L^{p}(J ; X)\right\}, \\
H_{\mu}^{1, p}(J ; X) & =\left\{u \in L_{\mu}^{p}(J ; X) \cap H_{l o c}^{1,1}(J ; X): u^{\prime} \in L_{\mu}^{p}(J ; X)\right\},
\end{aligned}
$$

Here $u^{\prime}$ denotes the time derivative of $u$ in the distributional sense. We aim for solutions in the maximal regularity space

$$
\mathbb{E}_{\mu}(J):=H_{\mu}^{1, p}\left(J ; X_{0}\right) \cap L_{\mu}^{p}\left(J ; X_{1}\right) .
$$

As space for the initial data $u_{0}$ we introduce the real interpolation space

$$
u_{0} \in X_{\gamma, \mu}=\left(X_{0}, X_{1}\right)_{\mu-1 / p, p} \text { and for } f \in \mathbb{F}_{\mu}(J):=L_{\mu}^{p}\left(J ; X_{0}\right),
$$

where $p \in(1, \infty)$. We define for $\beta \in[0,1]$ the space $X_{\beta}$ as the complex interpolation space $\left[X_{0}, X_{1}\right]_{\beta}$.

The following existence and uniqueness results are based on the following assumptions:

(H1) $\quad A$ has maximal $L^{p}$-regularity for $p \in(1, \infty)$.

(H2) $\quad F: X_{\beta} \rightarrow X_{0}$ satisfied the estimate

$$
\left\|F\left(u_{1}\right)-F\left(u_{2}\right)\right\|_{X_{0}} \leq C\left(\left\|u_{1}\right\|_{X_{\beta}}+\left\|u_{2}\right\|_{X_{\beta}}\right)\left(\left\|u_{1}-u_{2}\right\|_{X_{\beta}}\right)
$$

for some $C>0$ independent of $u_{1}, u_{2}$.

(H3) $2 \beta-1+1 / p \leq \mu$.

(S) $X_{0}$ is of class UMD, and the embedding

$$
H^{1, p}\left(\mathbb{R} ; X_{0}\right) \cap L^{p}\left(\mathbb{R} ; X_{1}\right) \hookrightarrow H^{1-\beta, q}\left(\mathbb{R} ; X_{\beta}\right)
$$

is valid for each $\beta \in(0,1)$ and $p \in(1, \infty)$.

For the definition and properties of UMD spaces see e.g. [12].

Proposition 2 [21, Theorem 1.2] Assume that the assumptions (H1), (H2), (H3) and (S) hold and let

$$
u_{0} \in X_{\gamma, \mu} \quad \text { and } f \in L_{\mu}^{p}\left(0, T ; X_{0}\right) .
$$

Then there exists a time $T^{\prime}=T^{\prime}\left(u_{0}, f\right)$ with $0<T^{\prime} \leq T$ such that problem (4.1) admits a unique solution

$$
u \in H_{\mu}^{1, p}\left(0, T^{\prime} ; X_{0}\right) \cap L_{\mu}^{p}\left(0, T^{\prime} ; X_{1}\right) .
$$

Furthermore, the solution u depends continuously on the data.

\section{Remark 4}

(a) We note that condition $(S)$ holds true whenever $X_{0}$ is of class UMD and there is an operator $A_{\#} \in \mathcal{H}^{\infty}\left(X_{0}\right)$ with domain $D\left(A_{\#}\right)=X_{1}$ satisfying $\phi_{A_{\#}}^{\infty}<\pi / 2$, see Remark 1.1 of [21].

(b) Due to the embeddings 


$$
\mathbb{E}_{\mu}\left(0, T^{\prime}\right) \hookrightarrow C\left(\left[0, T^{\prime}\right] ; X_{\gamma, \mu}\right) \quad \text { and } \quad \mathbb{E}_{\mu}\left(\delta, T^{\prime}\right) \hookrightarrow C\left(\left[\delta, T^{\prime}\right] ; X_{\gamma}\right), \quad \delta>0,
$$

there is an instantaneous smoothing effect typical for parabolic equations, compare e.g. [19, Section 3.5.2].

When investigating the question of a global solution, we consider

$$
t_{+}\left(u_{0}\right):=\sup \left\{T^{\prime}>0: \text { equation (4.1) admits a solution } v \in \mathbb{E}_{\mu}\left(0, T^{\prime}\right)\right\} .
$$

By the above Proposition 2, this set is non-empty, and we say that (4.1) has a global solution if for $f \in L_{\mu}^{q}\left(0, T ; X_{0}\right), 0<T<\infty$, which can be extended trivially to $(0, \infty)$, one has $t_{+}\left(u_{0}\right)=\infty$. Global existence results can be derived from suitable a priori bounds following [19, Theorem 5.7.1].

In the particular case of bilinear nonlinearities, i.e. $F(u)=G(u, u)$ with $G: X_{\beta} \times X_{\beta} \rightarrow$ $X_{0}$ bilinear and bounded one obtains a global solution to (4.1) provided the data are small enough in the $\left(X_{0}, X_{1}\right)_{1-1 / p, p}$-norm. More precisely, the following corollary to Proposition 2 holds.

Corollary 3 [20, Cor. 2.2] Let the assumptions of Proposition 2 hold and assume that $f=0$ and that $F(u)=G(u, u)$, where $G: X_{\beta} \times X_{\beta} \rightarrow X_{0}$ is bilinear and bounded. If $0 \in$ $\varrho(A)$ and $1 / p<1-\beta$, then the trivial solution to (4.1) is exponentially stable in $\left(X_{0}, X_{1}\right)_{1-1 / p, p}$.

Let now $\Omega \subset \mathbb{R}^{3}$ be a bounded and convex domain and suppose that $1<q \leq 2$. We rewrite the chemotaxis system (1.1) as a semilinear equation for $w=(u, v)^{T}$ of the form

$$
\left\{\begin{array}{c}
w^{\prime}(t)+\mathcal{A} w(t)=F(w(t)), \quad t \in(0, T), \\
w(0)=w_{0}
\end{array}\right.
$$

in $X_{0}:=W^{-1, q}(\Omega) \times L^{q}(\Omega)$. Here $\mathcal{A}$ on $X_{0}$ is defined by

$$
\mathcal{A}:=-\left(\begin{array}{cc}
\Delta_{N}^{w} & 0 \\
1 & \Delta_{N}-1
\end{array}\right),
$$

where $\Delta_{N}^{w}$ with domain $D\left(\Delta_{N}^{w}\right)=W^{1, q}(\Omega)$ denotes the Neumann Laplacian on $\left(W^{1, q^{\prime}}(\Omega)\right)^{\prime}$ and $\Delta_{N}$ with domain $D\left(\Delta_{N}\right)=W_{N}^{2, q}(\Omega)=\left\{u \in W^{2, q}(\Omega): \partial_{v} u=0\right.$ on $\left.\partial \Omega\right\}$ denotes the Neumann Laplacian on $L^{q}(\Omega)$. Hence $X_{1}=D\left(\Delta_{N}^{w}\right) \times D\left(\Delta_{N}\right)$. Furthermore $F$ is given by

$$
F(w):=\left(\begin{array}{c}
-\nabla \cdot(u \nabla v) \\
0
\end{array}\right),
$$

In order to estimate the nonlinearity $F$ we use Hölder's inequality to obtain

$$
\begin{aligned}
\left\|F\left(w_{1}\right)-F\left(w_{2}\right)\right\|_{X_{0}} & \leq\left\|\operatorname{div}\left(u_{1} \nabla v_{1}\right)-\operatorname{div}\left(u_{2} \nabla v_{2}\right)\right\|_{X_{0}} \\
& \leq C\left\|\left(u_{1}-u_{2}\right) \nabla v_{1}\right\|_{L^{q}}+\left\|u_{2} \nabla\left(v_{1}-v_{2}\right)\right\|_{L^{q}} \\
& \leq C\left\|u_{1}-u_{2}\right\|_{L^{\prime} q}\left\|v_{1}\right\|_{H^{1, r q}}+\left\|u_{2}\right\|_{L^{r^{\prime} q}}\left\|v_{1}-v_{2}\right\|_{H^{1, r q}}
\end{aligned}
$$

with $1 / r+1 / r^{\prime}=1$ and $r^{\prime}, r>1$. We note that $X_{\beta}=X_{\beta}^{1} \times X_{\beta}^{2}$, where $X_{\beta}^{1}=H^{2 \beta-1, q}(\Omega)$ and $X_{\beta}^{2}=\left\{u \in H^{2 \beta, q}(\Omega): \partial_{v} u=0\right.$ on $\left.\Omega\right\}$ if $\beta \in(1 / 2+1 / 2 q, 1]$ and $X_{\beta}^{2}=H^{2 \beta, q}(\Omega)$ if 
$\beta \in[0,1 / 2+1 / 2 q)$. Hence, employing Sobolev's inequalities (see Theorem 4.12 of [1] for non-smooth domains) we obtain

$$
X_{\beta}^{1} \hookrightarrow H^{2 \beta-1, q}(\Omega) \hookrightarrow L^{r^{\prime} q}(\Omega) \text { and } X_{\beta}^{2} \hookrightarrow H^{2 \beta, q}(\Omega) \hookrightarrow H^{1, r q}(\Omega)
$$

provided $2 \beta-1-3 / q=-3 / q r^{\prime}$ and $2 \beta-3 / q=1-3 / q r$. This yields $\beta=(2+3 / q) / 4$. The condition $\beta<1$ requires thus the condition $q>3 / 2$. Condition (H3) requires $2 \beta \leq 1+$ $\mu-1 / p$ and the optimal choice for $\mu=\mu_{c}$ is $\mu_{c}=3 / 2 q+1 / p$. The condition $\mu \leq 1$ requires then the condition $3 / 2 q+1 / p \leq 1$. We then obtain

$$
\begin{aligned}
\left\|F\left(w_{1}\right)-F\left(w_{2}\right)\right\|_{X_{0}} & \leq C\left(\left\|\left(u_{1}-u_{2}\right)\right\|_{X_{\beta}^{1}}\left\|v_{1}\right\|_{X_{\beta}^{2}}+\left\|u_{2}\right\|_{X_{\beta}^{1}}\left\|\left(v_{1}-v_{2}\right)\right\|_{X_{\beta}^{2}}\right) \\
& \leq C\left(\left\|w_{1}\right\|_{X_{\beta}}+\left\|w_{2}\right\|_{X_{\beta}}\right)\left\|w_{1}-w_{2}\right\|_{X_{\beta}} .
\end{aligned}
$$

Summarizing, note that assumptions (H1) and (S) of Proposition 2 are satisfied due to assertions (a), (b) and (d) of Proposition 1 and Remark 3. The above calculations show that also the assumptions (H2) and (H3) are satisfied. We thus conclude the assertion of Theorem 1 by Proposition 2.

The assertion of Corollary 1 follows from Corollary 3.

\section{Strong time periodic solutions}

We start by recalling from [4] that time periodic solutions to linear Cauchy problems within the maximal $L^{p}$-regularity class are well understood. Indeed, let $X_{0}$ be a Banach space and $A: X_{1} \rightarrow X_{0}$ be a linear operator. For $p \in(1, \infty)$ and setting

$$
\mathbb{F}:=L^{p}\left(0,2 \pi ; X_{0}\right), \quad \mathbb{E}:=H_{p}^{1}\left(0,2 \pi ; X_{0}\right) \cap L^{p}\left(0,2 \pi ; X_{1}\right),
$$

we say that $A$ admits maximal periodic $L^{p}$-regularity if for each $f \in \mathbb{F}$ there is a unique solution $u \in \mathbb{E}$ to

$$
\left\{\begin{array}{l}
u^{\prime}(t)+A u(t)=f(t), \quad t \in(0,2 \pi), \\
u(0)=u(2 \pi)
\end{array}\right.
$$

In this case, by the closed graph theorem, there exists a constant $M>0$ satisfying $\|u\|_{\mathbb{E}} \leq M\|f\|_{\mathbb{F}}$. Maximal periodic $L^{p}$-regularity has been characterized by Arendt and $\mathrm{Bu}$ [4] in terms of the $\mathcal{R}$-boundedness of the resolvent of $A$ on a suitable subset of $\mathbb{C}$. Their result reads as follows.

Proposition 3 [4, Theorem 2.3] Let $1<p<\infty, X$ be a UMD-space and A be a closed operator in $X$. Then the following assertions are equivalent.

a) A admits maximal periodic $L^{p}$-regularity.

b) $i \mathbb{Z} \subset \varrho(-A)$ and $\left.\left(k(i k+A)^{-1}\right)\right)_{k \in \mathbb{Z}}$ is $\mathcal{R}$-bounded.

For definitions of UMD-spaces and $\mathcal{R}$-bounded families of operators as well as their properties, we refer to $[5,12,19]$. The relationship between maximal periodic $L^{p}$-regularity and maximal $L^{p}$-regularity for the Cauchy problem can be described, following again the results of Arendt and $\mathrm{Bu}$ [4], as follows. 
Proposition 4 [4, Thm. 5.1] Let $X$ be a Banach space and $-A: D(A) \rightarrow X$ be the generator of a $C_{0}$-semigroup on $X$. Then $A$ admits maximal periodic $L^{p}$-regularity if and only if $1 \in \rho\left(e^{-2 \pi A}\right)$ and $A$ admits maximal $L^{p}$-regularity, i.e., if for each $f \in \mathbb{F}$ there is a unique solution $u \in \mathbb{E}$ to

$$
\left\{\begin{array}{l}
u^{\prime}(t)+A u(t)=f(t), \quad t \in(0,2 \pi) \\
u(0)=0
\end{array}\right.
$$

An extension of the above result to the quasilinear and semilinear situation was given in [8]. To formulate this result, let $X_{0}, X_{1}$ be Banach spaces with $X_{1}$ densely embedded in $X_{0}$ and for $1<p<\infty$ set $X_{\gamma}=\left(X_{0}, X_{1}\right)_{1-1 / p, p}$. We introduce the following Lipschitz condition on the nonlinearity $F$.

(L) Let $F:[0,2 \pi] \times X_{\gamma} \rightarrow X_{0}$ satisfy $F(\cdot, v(\cdot)) \in \mathbb{F}$ for all $v \in \mathbb{E}$ and suppose that for each $R>0$ there exists $C(R)>0$ such that

$$
\|F(\cdot, v(\cdot))-F(\cdot, w(\cdot))\|_{\mathbb{F}} \leq C(R)\|v-w\|_{\mathbb{E}}
$$

for all $v, w \in \overline{B_{\mathbb{E}}}(0, R)$.

Proposition 5 [8, Cor. 3.5] Let $X_{0}, X_{1}$ be Banach spaces with $X_{1}$ densely embedded in $X_{0}$ and let $A: X_{1} \rightarrow X_{0}$ be a closed linear operator satisfying maximal periodic $L^{p}$-regularity for $p \in(1, \infty)$. Assume furthermore that (L) is satisfied. There is $\delta_{1}>0$ such that, if $C(R)<\delta_{1}$ for some $R>0$, then there are $\delta_{2}>0$ and $r>0$ such that if $\|F(\cdot, 0)\|_{\mathbb{F}}<\delta_{2}$ there is a unique solution $u \in \overline{B_{\mathbb{E}}}(0, r)$ to

$$
\left\{\begin{array}{l}
u^{\prime}(t)+A u(t)=F(t, u(t)), \quad t \in(0,2 \pi), \\
u(0)=u(2 \pi) .
\end{array}\right.
$$

To prove Theorem 2 we note first that $-A_{0}$ on $X_{0}$ defined as in (2.3) admits maximal periodic $L^{p}$-regularity on $X_{0}$. In fact, we have $0 \in \rho\left(\Delta_{N, 0}^{w}\right)$ and $0 \in \rho\left(\Delta_{N}-1\right)$ and hence $0 \in \rho\left(-A_{0}\right)$. Due to Corollary $2,-\Delta_{N, 0}^{w}$ on $\left(W^{1, q^{\prime}}(\Omega) \cap L_{0}^{q^{\prime}}(\Omega)\right)^{\prime}$ as well as $-\left(\Delta_{N}-1\right)$ on $L^{q}(\Omega)$ admit maximal $L^{p}$-regularity. Hence, the triangular structure of $A_{0}$ implies that $-A_{0}$ admits maximal periodic $L^{p}$-regularity on $X_{0}$.

Next, we show that $H(\cdot, w(\cdot)) \in \mathbb{E}$ is satisfied for $w \in \mathbb{E}$. By Hölder's inequality

$$
\begin{aligned}
\|H(\cdot, w(\cdot))\|_{\mathbb{F}} & \leq c\|u \nabla v\|_{L^{p}\left(0, T ; L^{q}(\Omega)\right)}+\|f\|_{\mathbb{F}} \\
& \leq c\|u\|_{L^{2 p}\left(0, T ; L^{2 q}(\Omega)\right)}\|v\|_{L^{2 p}\left(0, T ; W^{1,2 q}(\Omega)\right)}+\|f\|_{\mathbb{F}} .
\end{aligned}
$$

In view of the assumption $\frac{3}{2}<q \leq 2$ and $\frac{1}{p}+\frac{3}{2 q}<1$ we may choose $\theta \in(0,1)$ satisfying $\frac{1}{2 p}<\theta<\frac{1}{2}-\frac{3}{4 q}$. The mixed derivate theorem and Sobolev estimates for convex domains (see e.g. [1] Thm. 4.12) yield 


$$
\begin{aligned}
\|H(\cdot, w(\cdot))\|_{\mathbb{F}} & \leq c\|u\|_{H^{\theta, p}\left(0, T ; H^{-1+2(1-\theta), q}(\Omega)\right)}\|v\|_{H^{\theta, p}\left(0, T ; H^{2(1-\theta), q}(\Omega)\right)}+\|f\|_{\mathbb{F}} \\
& \leq c\|w\|_{\mathbb{E}}^{2}+\|f\|_{\mathbb{F}} .
\end{aligned}
$$

Furthermore, since $\left(W^{1, q^{\prime}}(\Omega)\right)^{\prime} \subset\left(W^{1, q^{\prime}}(\Omega) \cap L_{0}^{q^{\prime}}(\Omega)\right)^{\prime}$, we see that the first component of $H$ belongs to $L^{p}\left(0, T ;\left(W^{1, q^{\prime}}(\Omega) \cap L_{0}^{q^{\prime}}(\Omega)\right)^{\prime}\right)$. Hence, $H(\cdot, w(\cdot)) \in \mathbb{F}$. In order to verify condition (L), let $w, \tilde{w} \in \overline{B_{\mathbb{E}}}(0, R)$. By Hölder's inequality, the mixed derivative theorem and Sobolev's inequality we obtain

$$
\begin{aligned}
\| H(\cdot, w(\cdot))- & H(\cdot, \tilde{w}(\cdot)) \|_{\mathbb{F}} \\
\leq & c\left(\|u-\tilde{u}\|_{L^{2 p}\left(0, T ; L^{2 q}(\Omega)\right)}\|v\|_{L^{2 p}\left(0, T ; W^{1,2 q}(\Omega)\right)}\right. \\
& \left.+\|\tilde{u}\|_{L^{2 p}\left(0, T ; L^{2 q}(\Omega)\right)}\|v-\tilde{v}\|_{L^{2 p}\left(0, T ; W^{1,2 q}(\Omega)\right)}\right) \\
\leq & c\left(\|u-\tilde{u}\|_{H^{\theta, p}\left(0, T ; H^{-1+2(1-\theta), q}(\Omega)\right)}\|v\|_{H^{\theta \theta p}\left(0, T ; H^{2(1-\theta), q}(\Omega)\right)}\right. \\
& \left.+\|\tilde{u}\|_{H^{\theta, p}\left(0, T ; H^{-1+2(1-\theta), q}(\Omega)\right)}\|v-\tilde{v}\|_{H^{\theta, p}\left(0, T ; H^{2(1-\theta), q}(\Omega)\right)}\right) \\
\leq & c\left(\|u-\tilde{u}\|_{\mathbb{E}_{1}}\|v\|_{\mathbb{E}_{2}}+\|\tilde{u}\|_{\mathbb{E}_{1}}\|v-\tilde{v}\|_{\mathbb{E}_{2}}\right) \\
\leq & c\left(\|w\|_{\mathbb{E}}+\|\tilde{w}\|_{\mathbb{E}}\right)\|w-\tilde{w}\|_{\mathbb{E}} \\
\leq & c R\|w-\tilde{w}\|_{\mathbb{E}} \cdot
\end{aligned}
$$

The proof of Theorem 2 is complete.

Funding Open Access funding enabled and organized by Projekt DEAL.

\section{Declarations}

Conflict of interest The authors declare that they have no conflict of interest.

Open Access This article is licensed under a Creative Commons Attribution 4.0 International License, which permits use, sharing, adaptation, distribution and reproduction in any medium or format, as long as you give appropriate credit to the original author(s) and the source, provide a link to the Creative Commons licence, and indicate if changes were made. The images or other third party material in this article are included in the article's Creative Commons licence, unless indicated otherwise in a credit line to the material. If material is not included in the article's Creative Commons licence and your intended use is not permitted by statutory regulation or exceeds the permitted use, you will need to obtain permission directly from the copyright holder. To view a copy of this licence, visit http://creativecommons.org/licenses/by/4.0/.

\section{References}

1. Adams, R.A., Fournier, J.J.F.: Sobolev Spaces. Academic Press, Amsterdam (2003)

2. Amann, H.: Nonhomogeneous linear and quasilinear elliptic and parabolic boundary value problems. In: H.J. Schmeisser, H. Triebel (eds.), Function Spaces, Differential Operators and Nonlinear Analysis, Teubner, pp. 9-126 (1993)

3. Amann, H.: Linear and Quasilinear Parabolic Problems, Monographs in Mathematics, Vol. 89, Birkhäuser (1995)

4. Arendt, W., Bu, S.: The operator-valued Marcinkiewicz multiplier theorem and maximal regularity. Math. Z. 240, 311-343 (2002)

5. Denk, R., Hieber, M., Prüss, J.: $\mathcal{R}$-Boundedness, Fourier multipliers and problems of elliptic and parabolic type. Mem Am. Math. Soc. 788 (2003)

6. Hieber, M., Prüss, J.: Functional calculi for linear operators in vector-valued $L^{p}$-spaces via the transference principle. Adv. Differ. Equ. 3, 847-872 (1998) 
7. Hieber, M., Robinson, J., Shibata, Y.: Mathematical Analysis of the Navier-Stokes Equations. Lecture Notes in Mathematics, vol. 2254. CIME Found. Subseries, Springer, Cham (2020)

8. Hieber, M., Stinner, C.: Strong time periodic solutions to Keller-Segel systems: an approach by the quasilinear Arendt-Bu theorem. J. Differ. Equ. 269, 1636-1655 (2020)

9. Hillen, T., Painter, K.J.: A user's guide to PDE models for chemotaxis. J. Math. Biol. 58, 183-217 (2009)

10. Horstmann, D.: From 1970 until present: the Keller-Segel model in chemotaxis and its consequences I. Jahresber. Deutsch. Math.-Verein. 105(2003), 103-165

11. Horstmann, D., Meinlschmidt, H., Rehberg, J.: The full Keller-Segel model is well-posed on nonsmooth domains. Nonlinearity 31, 1560-1592 (2018)

12. Hytönen, T., van Neerven, J., Veraar, M., Weis, L.: Analysis in Banach Spaces, Vol. II. Springer, New York (2017)

13. Kozono, H., Sugiyama, Y.: Global strong solution to the semi-linear Keller-Segel system of parabolicparabolic type with small data in scale invariant spaces. J. Differ. Equ. 247, 1-32 (2009)

14. Kozono, H., Sugiyama, Y.: Strong solutions to the Keller-Segel system with the weak $L^{n / 2}$ initial data and its application to the blow-up rate. Math. Nachr. 283, 732-751 (2010)

15. Kozono, H., Sugiyama, Y., Wachi, T.: Existence and uniqueness theorem on mild solutions to the Keller-Segel system in the scaling invariant space. J. Differ. Equ. 252, 1213-1228 (2012)

16. Langkeit, J., Winkler, M.: Facing low regularity in chemotaxis systems. Jahresber. Deutsch. Math.Verein. 122, 35-64 (2020)

17. Nakaguchi, E., Osaki, K.: $L_{p}$-estimates of solutions to $n$-dimensional parabolic-parabolic system for chemotaxis with subquadratic degradation. Funkcial. Ekvac. 59, 51-66 (2016)

18. Nakaguchi, E., Osaki, K.: Global existence of solutions to an $n$-dimensional parabolic-parabolic system for chemotaxis with logistic-type growth and superlinear production. Osaka J. Math. 55, 51-70 (2018)

19. Prüss, J., Simonett, G.: Moving Interfaces and Quasilinear Parabolic Evolution Equations, Monographs in Mathematics, Vol. 105, Birkhäuser (2016)

20. Prüss, J., Simonett, G., Wilke, M.: Criticial spaces for quasilinear parabolic evolution equations and applications. J. Differ. Equ. 264, 2028-2074 (2018)

21. Prüss, J., Wilke, M.: Addendum to the paper "On quasilinear parabolic evolution equations in weighted $L_{p}$-spaces II". J. Evol. Equ. 17, 1381-1388 (2017)

22. Tao, Y.: Global dynamics in a higher dimensional repulsion chemotaxis model with nonlinear sensitivity. Discrete Contin. Dyn. Syst. Ser. B 18, 2705-2722 (2013)

23. Tao, Y., Winkler, M.: Boundedness in a quasilinear parabolic-parabolic Keller-Segel system with subcritical sensitivity. J. Differ. Equ. 252, 692-715 (2012)

24. Wood, I.: Maximal $L^{p}$-regularity for the Laplacian on Lipschitz domains. Math. Z. 255, 855-875 (2007) 